\title{
Adaptive Control Topology based Optimization of Wind Power Dispatch
}

\author{
Adla Vinod and Ashoke Kumar Sinha \\ National Institute of Technology Silchar, INDIA, \\ disisvinnu@gmail.com, ashokeisinha2001@yahoo.com
}

\begin{abstract}
In this paper Optimized Zero-Phase Low-Pass Filter (OZPLPF) based wind power dispatch optimization with an Energy Storage System (ESS). The novelty of the proposed method is Artificial Bees Colony (ABC) algorithm based filter coefficients optimization. Because, the conventional filters are works with the non-adaptive filter coefficients, which requires larger capacity of the ESS. Here, the ABC algorithm is finds the filter coefficients for various wind power generations. In order to achieve the adaptive coefficients Signal to Noise Ratio (SNR) is consider as the objective function. By using the optimized coefficients, the delay response has been eliminated and significantly reduces the capacity of the ESS. The proposed method is implemented in the MATLAB/simulink platform and the effectiveness of the method is verified by comparison with the conventional methods. The comparison results were proved the superiority of the proposed method.
\end{abstract}

Keywords: ABC, OZPLPF, power dispatch, SNR, ESS, filter coefficients

\section{Introduction}

Wind Power Generation Systems (WPGSs) are used widespread equally with autonomous systems pertaining to energy supplying out of the remote loads and with grid-connected applications [1]. Picking particular turbine power generator engineering is usually a significant layout aspect in WPGSs [2]. Generally speaking, adjustable speed turbine power generator technologies usually are favored in comparison with continual speed turbines while they provide better voltage and rate of recurrence regulations and crop the ideal energy by current atmospheric attributes [3]. An electrical power generator could be often the squirrel cage induction generator (SCIG) or perhaps the doubly fed induction generator (DFIGURE) or the synchronous generators (SG) [4]. The electrical power generation by WPGSs is usually managed with two locations: variable power production and constant power production [5] [6]. The constant power production location is usually initialized in the event of the wind speed moves rated speed and becomes deactivate if it grows to further than cut-off value of which, power generator device can't carry the turbine shaft speed. In addition to adjustable energy technology is usually initialized in the event when the wind speed is usually involving rated value and cut-in value which can be the minimum value pertaining to WPGSs to begin a new technology [7].

In this particular area, for unique value associated with wind flow velocity, the grabbed wind flow energy can be a function associated with rotor velocity in support of in the event that most of us operate the rotor on optimum velocity may extract maximum energy from the wind flow [8]. Thus, the maximum power point tracking (MPPT) process is essential for pertaining WPGSs [9]. The particular MPPT formula can be a formula which often raises the power conversion efficiency of mechanical to electrical by simply controlling the turbine rotor velocity as outlined by actual wind flow speeds [10]. Nevertheless, the WG energy output can be mechanically controlled by simply altering the blade pitch angle [11]. And it is not invariably cost effective to accomplish, and so the high-efficiency energy conversion rate so that, these components extracts the absolute maximum power from the wind flow required therefore likewise further minimizes the overall system's cost [12]. The particular MPPT methods usually are primarily is dependent upon wind flow velocity calculations/estimations,

Received: March $3^{\text {th }}, 2017$. Accepted: Desember $30^{\text {th }}, 2018$ 
output power measurement/calculation and characteristic power curve [13]. They're used in control systems like tip speed ratio (TSR), the optimal torque (OT), the power mapping and the perturbation \& observation (P\&O)/hill climbing searching (HCS) [14].

TSR requires wind speed and rotor speed calculations which in turn raises the implementation in addition to preservation expense although the power mapping control requires system turbine powers curve details vs. its speed which remains as a tedious work [15][16]. And also HCS would be the greater control program which in turn doesn't call for any kind of before expertise in the device which is certainly independent of the turbine, generator and wind characteristics however it has some drawbacks for speed-efficiency tradeoff and wrong directionality in addition to drastically changing wind conditions [17]. Lately, soft computing tactics constructed from artificial intelligence (AIs) and global search algorithms (GSAs) are utilized to further improve the robustness to program modeling and guidelines variations in addition to optimizing the dynamic and static speed control performance [18][19]. The soft computing techniques tactics utilized in MPPT control algorithms tend to be largely dependent on fuzzy logic (FL), neural networks (NN), adaptive neural fuzzy inference system (ANFIS), radial basis function neural network (RBFNN), particle swarm optimization (PSO), and so on [20].

This paper OZPLPF based wind power dispatch optimization with an Energy Storage System (ESS). Here, the ABC algorithm is finds the filter coefficients for various wind power generations. In order to achieve the adaptive coefficients Signal to Noise Ratio (SNR) is consider as the objective function. By using the optimized coefficients, the delay response has been eliminated and significantly reduces the capacity of the ESS. The rest of the paper organized as follows: the recent research works are analyzed in section 2 ; the proposed work brief explanation is explained in section 3; the suggested technique achievement results and the related discussions are given in section 4; and section 5 ends the paper.

\section{Recent Research Work: A Brief Review}

Several research analysis performs happen to be persisted in literary works which appears on power model control of wind energy system by soft computing techniques. Some of them assessed right here. Hany L Jabr et al. [21] have suggested some sort of vector control scheme determined by neuro-fuzzy gain scheduler to control the rotor side voltage supply converter that granted unbiased control of the generated active along with reactive power and also the rotor speed to be able to trail the absolute maximum wind power level through wind driven DFIGURE for the consequence involving saturation in equally main along with loss flux paths. This insight for every single neuro-fuzzy system has been the problem benefit involving power generator speed, active or maybe reactive power which in turn made the device design and style easier. This proposed system has been simulated along with approved through experimental research carried out with a research laboratory DFIGURE. The entire DFIGURE system functionality using the proposed neuro-fuzzy logic tuner has been in comparison with that using the conventional PI controllers along with offered the power generator speed reaction and also the stator along with rotor currents as well as the rotor voltages in respond to a rapid change in the wind speed.

The Meharrar et al. [22] have proposed an Adaptive Neuro-Fuzzy Inference System (ANFIS) regarding variable-speed wind-generator for you to monitor the maximum powerpoint. It had been used to estimate the optimal speed rotation with all the variance on the wind speed as the feedback. The actual wind energy conversion system (WECS) utilized a new permanent magnet synchronous generator (PMSG) attached to a new DC bus having a power converter and has been modeled employing $\mathrm{d}-\mathrm{q}$ rotor research body. The wind speed step model had been employed in the design period. The actual functionality on the WECS while using proposed ANFIS controller had been tested regarding fast wind speed variation along with by simulation effects revealed that this possibility of achieving highest power monitoring to the wind along with end result voltage regulation to the DC bus at the same time while using 
ANFIS controller. Additionally they proved the excellent answer along with robustness on the proposed management system by looking at with fuzzy logic model.

M Narayana et al. [23] have offered a generic MPPT control algorithm depending on an Adaptive Filter together with a Fuzzy Logic for small-scale WECSs. The suggested Adaptive Filtration system had been utilized to establish a signal which represents the particular aerodynamic power that accounted for that dynamic circumstances and system losses. This kind of signal had been utilized with the hill-climbing control approach dependent on FLA to monitor the particular optimum working factors on the system by taking into consideration about the qualitative variation on the measured result details, with no expertise in system characteristics features. Through the achieved outcomes demonstrated which the offered "generic" MPPT controller done a lot better than the particular wind sensor techniques.

Whei-Min Lin et al. [24] have got designed a optimal wind flow energy management system for MPPT by using a grey predictor to the preprocessor, a high-performance on the online training Elman neural network (ENN) which in turn derived the particular turbine speed to extract maximum power from wind flow. The actual connective loads from the increased ENN were educated in online by the back propagation learning algorithm together with delta adaptation law with varied learning rates. Below, the particular speed controller established the generator torque command utilizing a current control loop. The actual offered criteria could maintain the system balance together with greater dynamic characteristics and achieved the required overall performance despite the presence of parameter concerns. The suggested method ended up applied in a real-time application, having a commercial IG and a dc drive, which in turn emulated the particular wind turbine actions. From the fresh benefits shown that the offered MPPT depending on grey ENN could gain large accuracy and reliability within the both trajectory prediction and target tracking.

Ali Michael Eltamaly et al. [25] have presented FLC for grid-connected WECS applying PMSG to monitor the maximum power for wind speed under wind turbine and ensured the energy would not go over the rated power for the over rated wind speeds. The wind turbine was attached to the grid via back-to-back PWM-VSC. In the planned process, the generator area operator was used to monitor the utmost power through preventing the rotational speed of the wind turbine applying FLC while grid area converter was for productive and reactive power get a handle on by preventing $\mathrm{q}$-axis and $\mathrm{d}$-axis recent parts respectively. Here, the d-axis recent was collection at zero for unity power element and the q-axis recent was controlled to provide the energy streaming from the dc-link to the electric utility grid.

Juan P.Torreglosa et al. [26] have presented a novel energy dispatching based on Model Predictive Control (MPC) for off-grid photovoltaic (PV)/wind turbine/hydrogen/battery hybrid systems. The renewable energy sources supply energy to the hybrid system and the battery and hydrogen system were used as energy storage devices. The denominated "hydrogen system" was composed of fuel cell, electrolyzer and hydrogen storage tank. The MPC generates the reference powers of the fuel cell and electrolyzer to satisfy different objectives: to track the load power demand and to keep the charge levels of the energy storage devices between their target margins. To show the proper operation of the proposed energy dispatching, a simpler strategy based on state control was presented in order to compare and validate the results for long-term simulations of 25 years (expected lifetime of the system) with a sample time of one hour.

Juan Pablo Saenz et al. [27] have discussed about Economic load dispatch (ELD) was the operation of generation plants producing reliable electricity at the lowest cost, while recognizing limitations of the system. The environmental economic load dispatch (EELD) problem extends the ELD to include environmental considerations which makes it even more challenging due to the fact that it considers a very large scale and dynamic system that was highly complex and has inherent uncertainty. They proposed a novel two-stage economic and environmental load dispatching framework using particle filtering for the efficient and reliable dynamic dispatching of electricity under uncertainty. Their proposed framework includes (1) a 
short term demand forecasting algorithm using wavelet transform adaptive modelling, and (2) a dynamic load dispatching algorithm using particle filtering developed in a simulation environment.

In variable pace drives, the significance of that algorithm is increased energy confine process at maximum power point over a broad variety of wind speeds. Also, the maximal aerodynamic efficiency is to attain by the use of optimum wind energy utilization with the help of the tracking controllers. Numerous control types of optimum wind power extraction methods are endured in literature which can be tip-speed tip speed ratio control, power signal feedback control, hill climb searching control and etc. But, these methods are inferior by the issue in the measurements of wind and turbine speed characteristics. Therefore, artificial intelligence techniques such as neural network and fuzzy are applied to resolve the estimation issue without using appropriate mathematical models. In neural network, the tracking is performed by the traditional data and the data are obtained from the behavior of the system. For this reason, a behavior analysis design is necessary for extracting the training data from the system. Fuzzy logic is control the energy conversion process significantly below dynamic variation of system parameters. But, the uncertainty handling capability of those intelligence techniques is transformed by hybrid learning model that's fuzzy neural network. On one other hand, the major drawback of the traditional, fuzzy neural network is to aid the application form domain is limited to static issues because of their feed forward network structure. The proposed methodology is briefly explained in the following section 3 .

\section{Power Smoothing Control Model for WT with ESS}

The synthesis output obtained from wind power and the energy storage systems must have the potential to follow the variation rule of the power load. In addition, it should reduce the greater deviations between the relative load from the wind power and the reverse adjustment characteristics. While considering the capacity of the energy storage systems, it should be small enough as per the requirement with improved protection, so that the total expense also gets reduced. An increase in the energy storage systems can effectively decrease and smooth the active power output fluctuations from a WT. Here, the ESS capacity has been minimized by using the proposed OZPLPF that utilizes optimum filter coefficients based on the objective function. The optimal filter coefficients avoid the phase delay and give a fine smoothing time constant. The wind power generation system in combination with the ESS, which uses the proposed OZPLPF, is described in Figure 1.

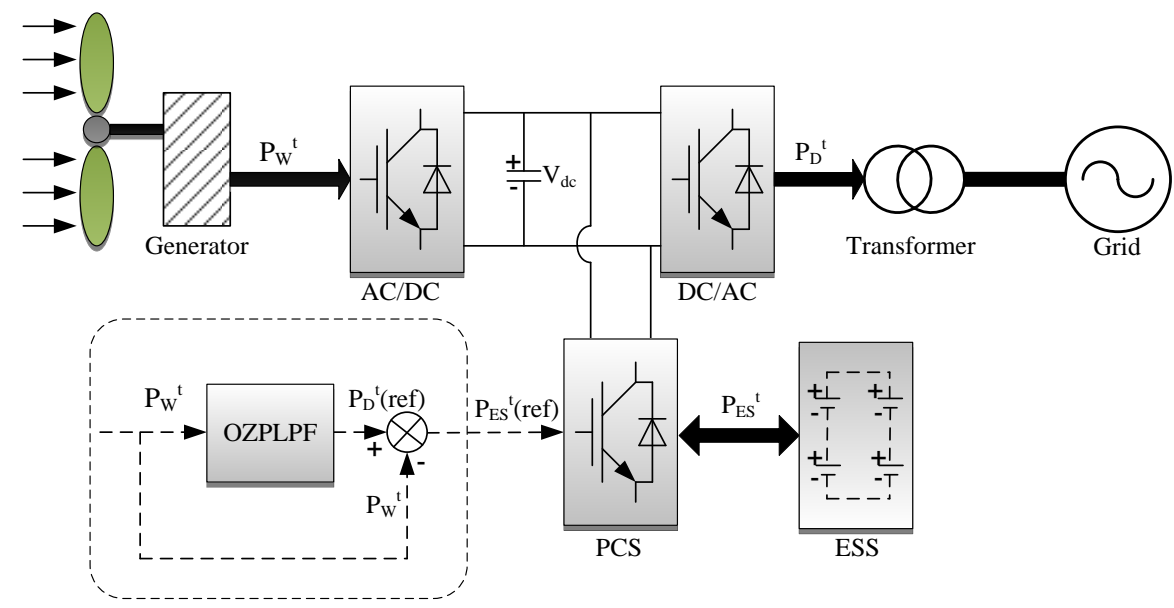

Figure 1. Proposed control model for WT and ESS

In Figure 1, the WT model is connected to the grid via the back to back converter model, i.e., rectifier and inverter. Several types of WT generators are available in the industry. The 
permanent magnet synchronous generator (PMSG) and the doubly fed induction generator (DFIGURE) are the familiar kinds among them [25,28]. The WT output power $P_{W T}^{t}$ is rectified into DC power and stored in the DC link capacitor. Here, the ESS is also connected through bidirectional DC-DC converter or Power Conversion system (PCS), which has the capability of delivering positive or negative power $P_{E S}^{t}$. Hence, the ESS can be charged or discharged using this type of connection. The stored energy in ESS can be released, when the dispatch power is less than the WT output power. Else, the surplus energy between the WT output power and dispatch power can be stored in the ESS. The output dispatch power is given to the grid through the transformer. The ESS effectively balances the variations in wind power and the grid is offered with an optimal power dispatch. Yet, having a massive ESS will not be cost-efficient and hence, it becomes necessary to use an ESS with minimum capacity. The minimum capacity ESS can be obtained, if there is an optimal power dispatch to the ESS. The optimal power dispatch can be accomplished by getting grid of the phase delay in the smoothing dispatch method. The output power of the ESS is given in equation (1), in which the power loss is neglected.

$$
P_{E S}^{t}=P_{D}^{t}-P_{W T}^{t}
$$

Here, $P_{E S}^{t}$ is the ESS power; $P_{W T}^{t}$ is the wind power captured by the wind turbine and $P_{D}^{t}$ is the dispatch power. The ESS power relies on both the power acquired from the WT $P_{W T}^{t}$ and the power dispatch $P_{D}^{t}$. Figure 1 clearly shows that the OZPLPF is utilized in the control scheme for deciding the power dispatch. The desired power of the ESS is nothing but the difference between the dispatch reference power and the wind turbine output power, which is described in equation (2).

$$
P_{E S}^{t}(r e f)=P_{D}^{t}(r e f)-P_{W T}^{t}
$$

$P_{E S}^{t}(r e f)$ indicate the desired power of ESS; $P_{D}^{t}(r e f)$ is the reference power dispatch. Here, the reference dispatch power is attained from the OZPLPF. The power dispatch of the WT is based on the ESS power charging and discharging, which is controlled by the PCS. If the PCS satisfies its reference, the power dispatch also tracks its reference successfully. This can be described in the following equations (3) and (4).

$$
\begin{aligned}
& P_{D}^{t}=P_{W T}^{t}+P_{E S}^{t} \\
& P_{D}^{t}=P_{W T}^{t}+\left[P_{D}^{t}(r e f)-P_{W T}^{t}\right]=P_{D}^{t}(r e f)
\end{aligned}
$$

Similarly, the power rating of the ESS and the rated energy of the ESS are described in the following equations (5), (6) and (7).

$$
\begin{aligned}
& P_{E S}(\text { rat })=\underset{0 \leq t \leq T}{\operatorname{Max}}\left|P_{E S}^{t}\right|=\operatorname{Max}\left|P_{D}^{t}-P_{W T}^{t}\right| \\
& E_{E S}^{t}=\int_{0}^{t} P_{E S}(\tau) d \tau=\int_{0}^{t}\left[P_{D}(\tau)-P_{W T}(\tau)\right] d \tau \\
& E_{E S}(\text { rat })=\underset{0 \leq t \leq T}{\operatorname{Max}}\left|E_{E S}^{t}\right|
\end{aligned}
$$

$E_{E S}^{t}$ is the net energy of the ESS; $T$ is the considered time interval; $P_{E S}(r a t)$ is the rated ESS output power and $E_{E S}(r a t)$ is the rated ESS energy. The proposed OZPLPF is briefly explained in section 3.2. 


\section{A. Power dispatch based on OZPLPF}

This section describes about the proposed OZPLPF model for power smoothing control of the WT in an ESS system. The literature review discusses about the weak points in the design and implementation of the dispatching methods. To overcome those problems, the OZPLPF is designed simply with a small number of coefficients. The OZPLPF incorporates the ABC optimization method for optimizing the filter coefficients $\left(\alpha_{k}\right)$. The $\mathrm{ABC}$ algorithm is a kind of swarm intelligence optimization algorithm, which imitates the food searching behaviour of the honey bees to produce solutions for numerical optimization problems [32] [33]. In $\mathrm{ABC}$, there are three phase in general. They are the employed bee phase, the onlooker bee phase and the scout bee phase. The reliability of $\mathrm{ABC}$ gets enhanced, if the food sources are effectively determined. Here, the ABC algorithm is used to optimize the filter coefficients based on the generated wind power output. The filter performance only decides the power dispatch and the capacity of the ESS. So, the ABC technique considers SNR as the objective function. The maximum SNR value reduces the noise of the signals [34]. The output power is optimally dispatched based on the outcome of the proposed filter. Initially, the gain parameters are randomly generated within the required limits, which are described in equation (8).

$$
\begin{aligned}
& X_{i}=\alpha_{i}^{\min } \leq \alpha_{i} \leq \alpha_{i}^{\max } \\
& \forall i=1,2 \ldots n
\end{aligned}
$$

$\alpha_{i}$ is the filter coefficient; the employed bee attains elite fitness function using equation (9) and the onlooker bee improves the velocity of the input populations using equation (10).

$$
\Phi=\text { Maximun }\left\{10 \log _{10} \frac{M A X^{2}}{M S E}\right\}
$$

MAX is the maximum possible value of the signal; $M S E$ is the mean square error between the actual signal and the noise signal. The equation for updation is described in equation (10).

$$
V_{i, j}=X_{i, j}+\Phi_{i, j}\left(X_{i, j}-X_{k, j}\right)
$$

$k=(1,2,3 \ldots n)$ and $j=(1,2,3 \ldots n)$ represent the randomly chosen indices. For the onlooker bee, the probability formula for selecting the nectar sources can be expressed as follows:

$$
\text { probability }=\frac{\Phi_{i}}{\sum_{i=1}^{n} \Phi_{i}}
$$

Discard the solutions, if they are not proven to be better and the random number for the scout bee is then generated by making use of equation (12).

$$
x_{i}^{j}=x_{\min }^{j}+\operatorname{rand}[0,1]\left(x_{\max }^{j}-x_{\min }^{j}\right)
$$

Then, the fine fitness for the new food sources are determined using equation (9) and the best solution refers to the optimal filter coefficients. The steps to find the optimal filter coefficients for power smoothing control using $\mathrm{ABC}$ algorithm are explained below.

\section{Steps of the ABC algorithm:}

Step 1: The entire parameters, which include the limits of the input parameters and the $\mathrm{N}$ limits of the random population, are initialized in this step of ABC algorithm. Here, the actual filter coefficient limit serves as the input.

Step 2: The filter coefficient parameters, $\alpha_{i}$, are randomly generated in accordance to the mentioned limits. This step comes under the employed bee phase. 
Step 3: The fitness of the random solutions can be obtained using equation (9) and the best solution can be discovered from this step of $\mathrm{ABC}$.

Step 4: In the onlooker bee phase, the neighborhood is searched. With this searching process, the velocity of the input population gets varied and this can be given by equation (10).

Step 5: In this step, the best solution sets and the worst solution sets are distinguished from one another.

Step 6: If there is a lack of best solution set from the onlooker bee phase, the solutions obtained from that phase is neglected and a random solution set for the scout bees is generated. This random generation can be done with equation (12).

Step 7: From each and every set of solutions, the best solution is computed and the fuel cost that is required for meeting up the load requirement is decided.

Step 8: The terminating condition is checked to know whether it has been fulfilled or not. If fulfilled, the process is ceased. Else, proceed to step 9.

Step 9: New solutions are then generated by allocating new population.

Once the above mentioned process has been finished, the $\mathrm{ABC}$ algorithm is ready to give the optimum coefficients for the ZPLPF. The output of the ABC algorithm is varied according to the variation in outcome of the WT. The ZPLPF with ABC algorithm structure is explained in Figure 3. The proposed technique is implemented in the MATLAB/Simulink platform and the results are analyzed in Section 4.

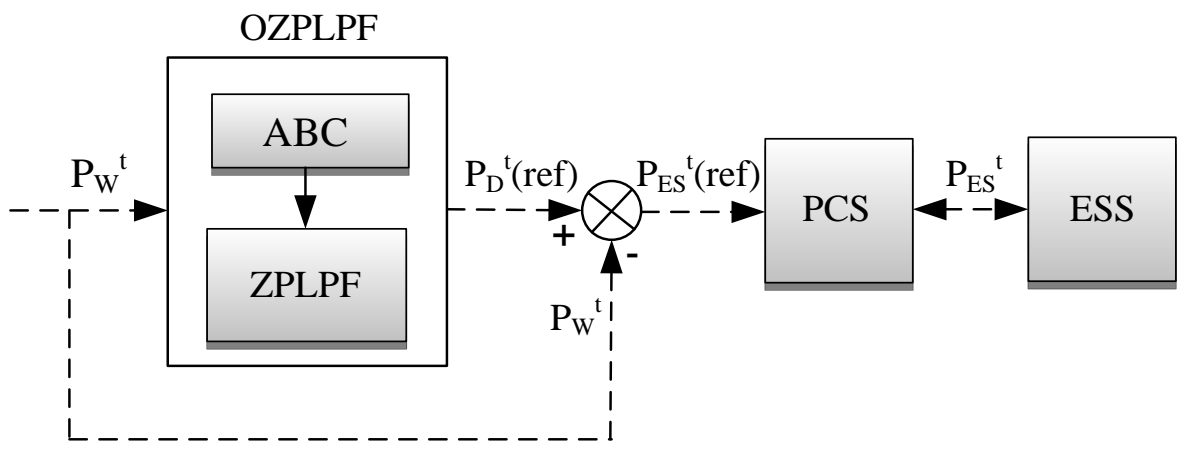

Figure 2. Structure of the OZPLPF

\section{Results and Discussion}

In this paper, adaptive control topology is proposed for ZPLPF based wind power dispatch with an ESS. Here, ABC algorithm is used to optimize the filter coefficients. By using the optimized coefficients, the delay response has been eliminated and reduced the capacity of the ESS. The proposed method is implemented in MATLAB/Simulink 7.10.0 (R2012a) working platform, 4GB RAM and Intel(R) core(TM) i5. Table 1 lists the various implementation parameters that have assisted in designing the test system. The proposed method is tested and their performances illustrated. 
Table 1. Implementation parameters

\begin{tabular}{|l|l|}
\hline Parameter & Value \\
\hline Stator resistance $\left(\mathrm{R}_{\mathrm{S}}\right)$ & $0.425 \Omega$ \\
\hline Stator inductance $\left(\mathrm{L}_{\mathrm{d}}\right.$ and $\left.\mathrm{L}_{\mathrm{q}}\right)$ & $0.01 \mathrm{mH}$ \\
\hline Flux induced by magnets $(\phi)$ & $0.433 \mathrm{wb}$ \\
\hline Moment of inertia $(\mathrm{J})$ & $0.0119 \mathrm{~kg} \mathrm{~m}^{2}$ \\
\hline Number of poles $(\mathrm{P})$ & 4 \\
\hline Switching frequency $\left(\mathrm{f}_{\mathrm{s}}\right)$ & $20 \mathrm{KHz}$ \\
\hline
\end{tabular}

\section{A. Performance analysis}

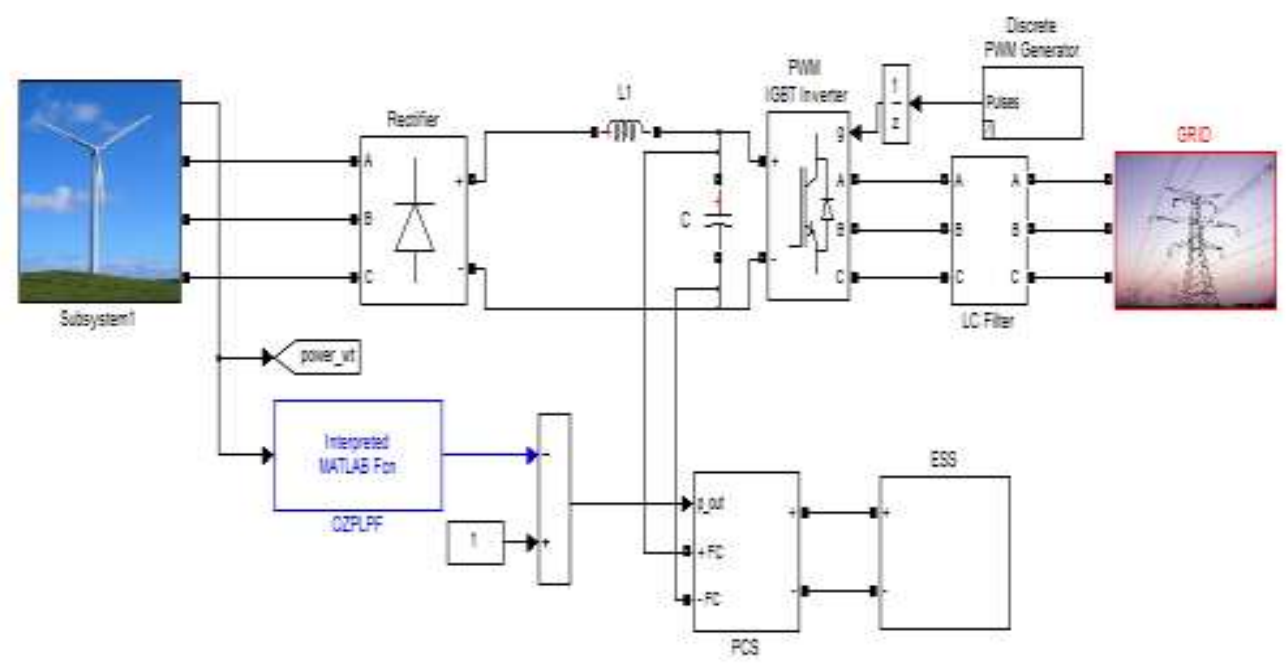

(a)

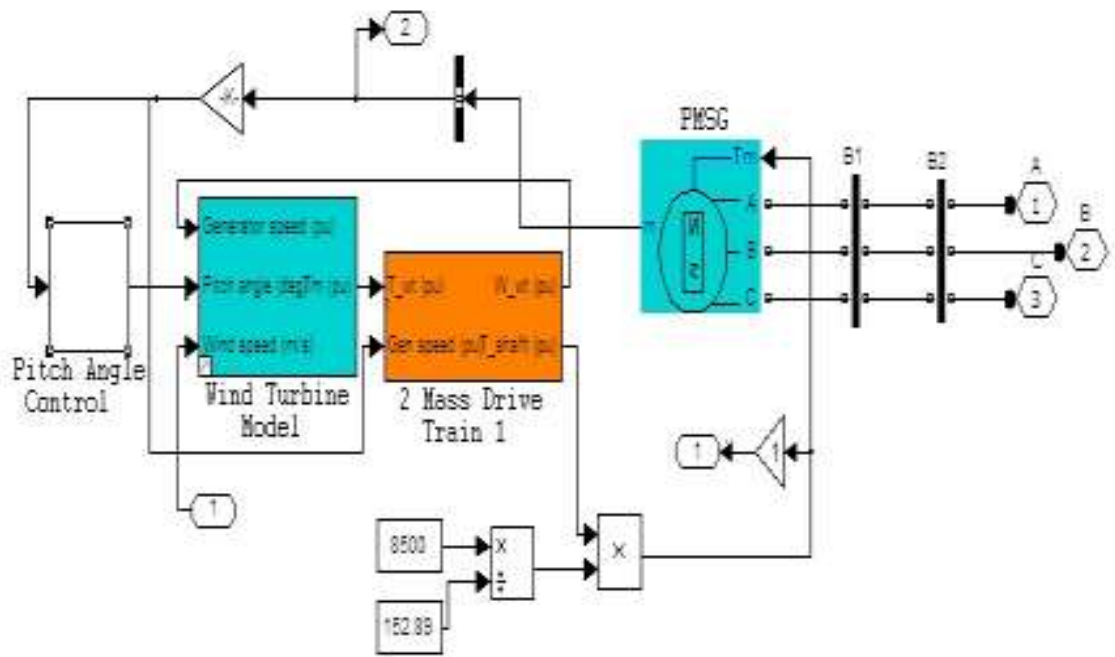

(b)

Figure 3. (a). Structure of the WT system with ESS using proposed method (b). Structure of the wind energy conversion system 


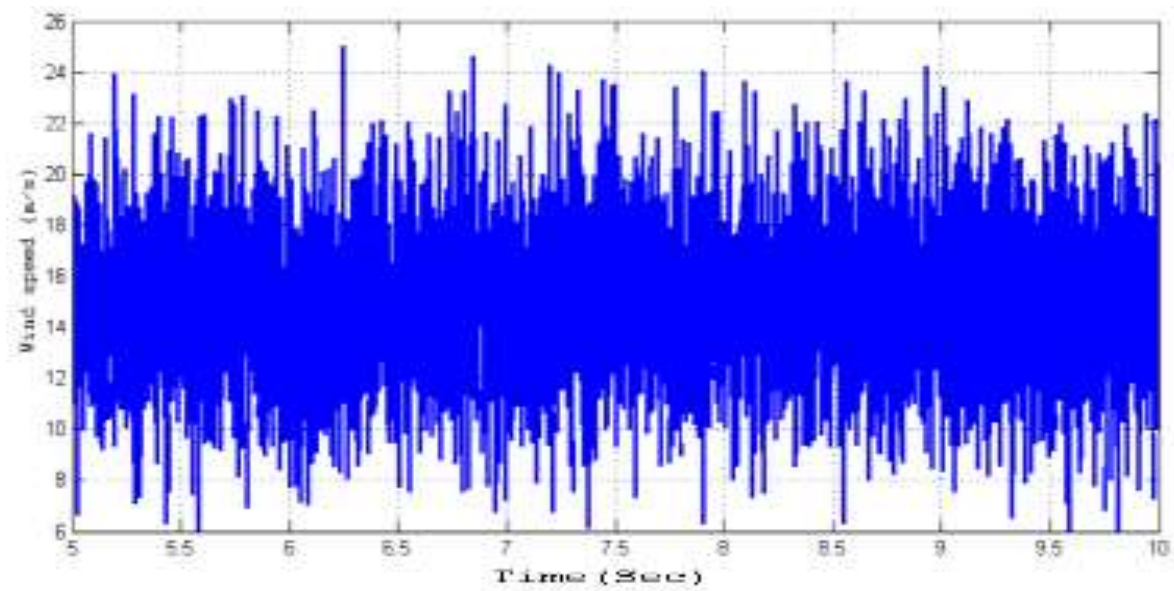

Figure 4. Wind speed profile

The wind speed profile which is used for obtaining the performance of several solution methodologies is depicted in Figure 4. In which the simulation time ranges from 5 to $10 \mathrm{sec}$ is illustrated in the Figure, but therefore $10 \mathrm{sec}$ of simulation time is used to identify the wind speed variation. The fluctuations in WT output power is enabled by ESS, which is caused due to the highly varying wind speed, is to be attenuated. Figure 4 shows power management of the proposed model for the change in wind speed. The power flow dispatch is used to determine the power and energy of the ESS. The wind speed attains a maximum speed of $25 \mathrm{~m} / \mathrm{s}$ at 6.35 sec and minimum speed at 5.6, 9.6 and $9.8 \mathrm{sec}$.

Figure 5 reveals the output power of the wind turbine and the power dispatch using Low Pass Filter (LPF). Fluctuations may arise while the output power is dispatched which depends on the variation in the input wind speed profile. It can be viewed that the variation in wind power dispatch is shown for 0.2 seconds to 0.3 seconds in Figure.5. Here, the WT output power is changed from $180 \mathrm{kWh}$ to $1550 \mathrm{kWh}$, denoting that there is fluctuation in the power dispatch. Moreover, utilization of LPF in the WT system with ESS causes phase delay problem, which in turn increases the capacity of ESS. The ESS capacity increases due to the fact that the output power delivered to the grid nearly equals the power generated from the WT and hence, demands a large-scale ESS that is more costly. So, it becomes highly essential to use a minimal-capacity ESS and to lessen the cost of the entire system.

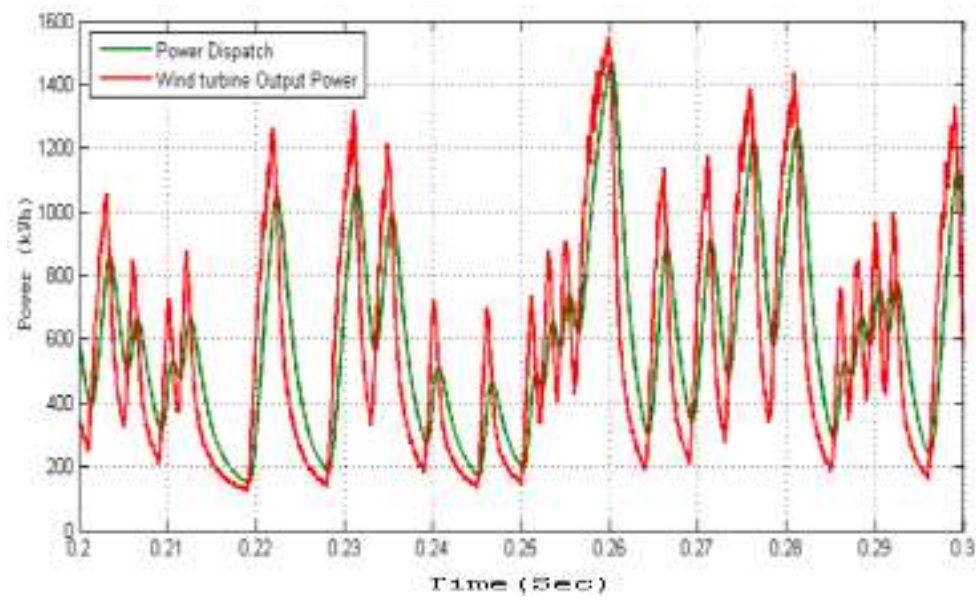

Figure 5. WT output power and output power delivered to the grid using LPF 


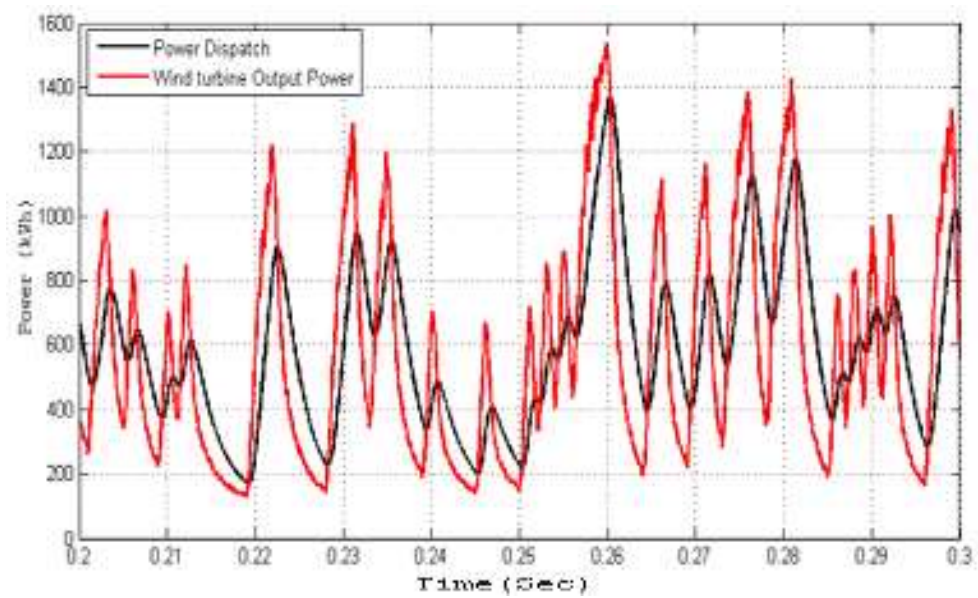

Figure 6. WT output power and output power delivered to the grid using ZPLPF

In Figure 6, the WT output power and the output power delivered to the grid using ZPLPF is shown. The ZPLPF is used to smooth the output power which is provided to the grid and so that the, power dispatch can be optimal. Yet it cannot help in adapting to the changing wind power conditions. It can be noted from the Figure that the ZPLPLF removes the large distortions in the output power given to the grid and in addition, it is more advantageous than that of the wind power dispatching system which make use of LPF, because the conventional ZPLPF has only a small number of coefficients and smoothing time constant. Using fixed values for the coefficients may cause problems. Therefore, the proposed algorithm is used to optimize the adaptive coefficients of the filter.

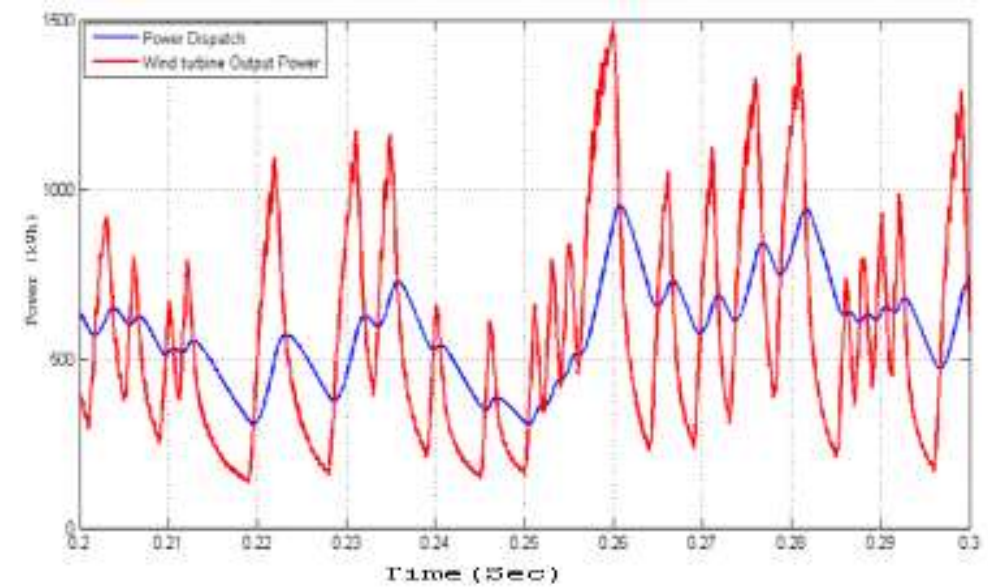

Figure 7. WT output power and output power delivered to the grid using proposed method

The performance of the ZPLPF and the filter quality was improved by using proposed method. In this paper, the filter is designed to achieve a simple computation. Therefore, the $\mathrm{ABC}$ optimization algorithm is utilized to optimize the adaptive coefficients of the filter. The objective function used in the ABC optimization algorithm is the SNR, which is obtained from various kinds of probable filter coefficients. Figure 7 show the WT output power and the output power delivered to the grid in the proposed scheme. In the time instant $t=0.255 \mathrm{sec}$, the wind turbine output power is highly increased than the dispatch power. Power evolution of the 
proposed system and maximum available power from the wind system is shown in above Figure. Consequently, the proposed method with OZPLPF is given to smooth the output power which is provided to the grid and so that the, power dispatch can be optimal. It is used to adopt for changing the wind power conditions. It can be noted from the Figure that the OZPLPF removes the large distortions in the output power given to the grid.

\section{B. Comparison Analysis}

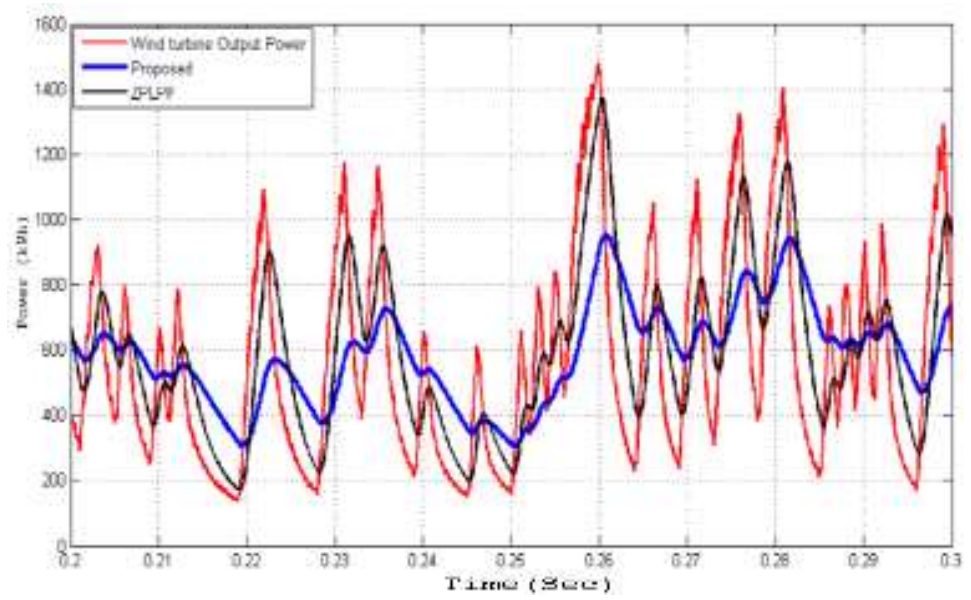

Figure 8. Comparison of WT output power and output power delivered to the grid

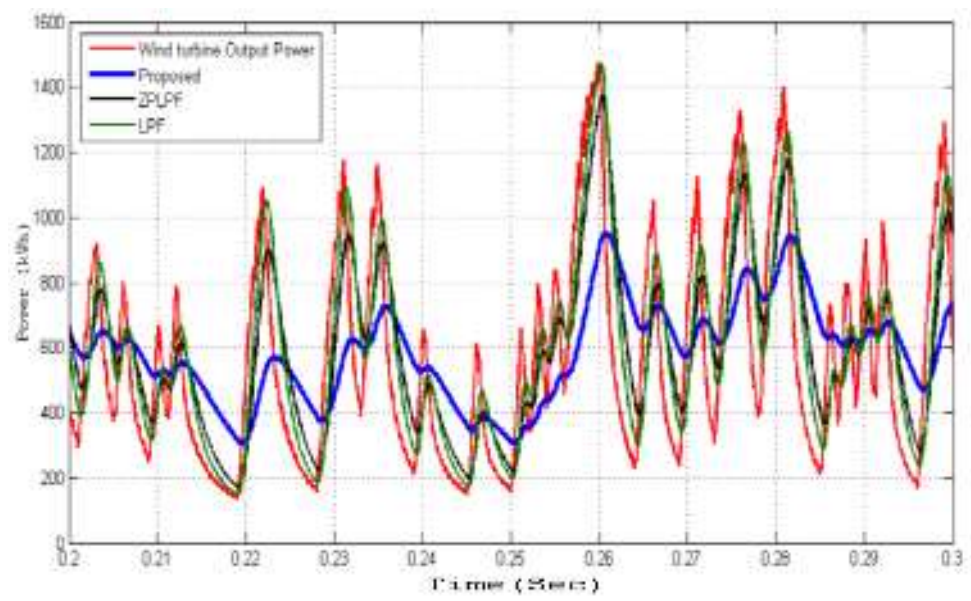

Figure 9. Comparison of WT output power and output power delivered to the grid

The performance of the WT power and output power is illustrated using the proposed and the existing method. The proposed method is compared against the systems which make use of a conventional ZPLPF and LPF in order to prove its effectiveness. The comparison results are shown in Figure 8 and Figure 9. It can be observed from these Figures that fluctuations are available in the WT output power in all the operating conditions between 0.2 to 0.3 seconds. In the LPF based dispatching system, phase lag is created in the LPF. So, that the response gets delayed and optimal power dispatch will not happen which leads to an increase in the ESS capacity. Next, the conventional ZPLPF has very few non-adaptive filter coefficients which cannot reduce the phase delay always and hence, the conventional ZPLPF cannot be 
appropriate for all wind speed variations. In the time instant $t=0.2$ to $t=0.3$, the performances of WT output power is noted and illustrated. In the Figure 8 and 9, the performances are noted in all the methods at every time seconds, the proposed method achieves the better performance compared with the above mentioned schemes because it has adaptive filter coefficients and it has the ability to reduce the phase delay problem as well as the ESS capacity. Similarly, the performances of the energy levels are depicted.

To evaluate the performances of the proposed and conventional ZPLPF methods, the responses of the energy levels are analyzed and are compared with the responses of the proposed method. The energy requirement in the proposed method and the conventional ZPLPF method are shown in Figure 10. Figure 11 deals with the ESS power requirement for the proposed method and the conventional ZPLPF method. In the Figure 10, the required energy levels are noted in the time instant $t=0.2$ to $t=0.3$ by using the proposed method. In the time instant $\mathrm{t}=0.2$, the proposed and existing energy levels are $350 \mathrm{kWh}$ and $600 \mathrm{kWh}$ are needed. When compared with other techniques, the minimum energy is needed for the proposed method. In the existing technique, the energy curve is initially increased at time instant $t=0.2$. From $t=0.203$, the energy curve starts to decrease and increase slowly and reaches the maximum energy $(850 \mathrm{kWh})$ after $\mathrm{t}=0.26 \mathrm{sec}$. While using the proposed technique, initially the curve reaches the energy at a low rate at $\mathrm{t}=0.2 \mathrm{sec}$. Then it is increased to $600 \mathrm{kWh}$ at $\mathrm{t}=0.26 \mathrm{sec}$. Again the curve starts to decrease and obtained its minimal energy of $280 \mathrm{kWh}$ at $\mathrm{t}=0.28 \mathrm{sec}$ even though there is a slight decrease at $\mathrm{t}=0.3 \mathrm{sec}$. In our proposed technique, the energy increases and decreases gradually from the beginning itself and reaches its minimum energy at $t=0.24 \mathrm{sec}$. On looking the minimum energy of the other techniques, the proposed method attained the lowest energy rate. Similarly, the performance of the power is also analyzed using various methods.

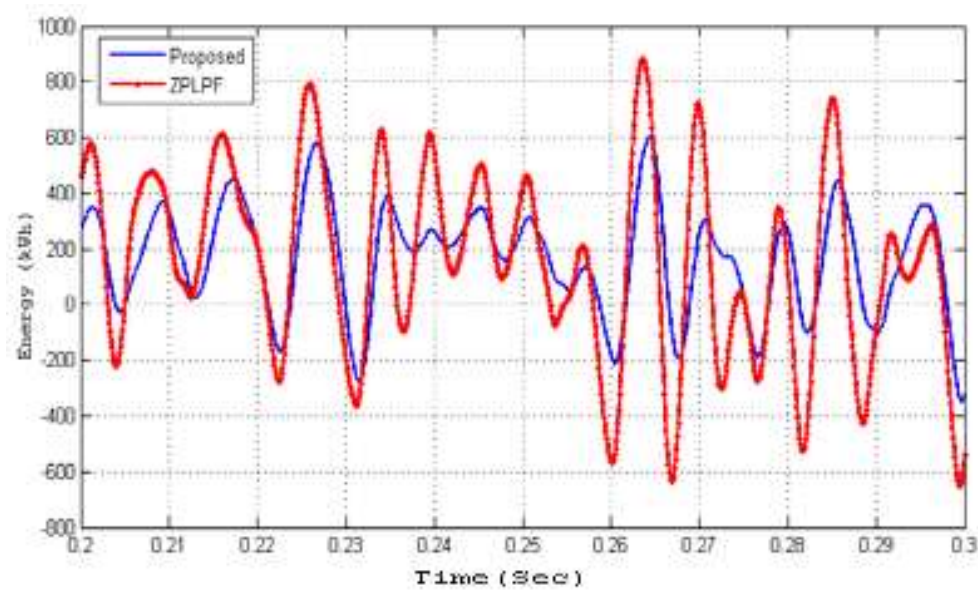

Figure 10. WT output power and output power delivered to the grid

By using conventional ZPLPF method, the performances of the WT output power is illustrated and compared with the proposed method. In the time instant $t=0.2$ to $t=0.3 \mathrm{sec}$, the power curve are illustrated and which shows that, it needs high power. In the proposed method, the WT output power increases and decreases gradually from the beginning itself and reaches its minimal power at $\mathrm{t}=0.24 \mathrm{sec}$. On looking the minimum power of the other techniques, the proposed method attained the lowest power rate. 


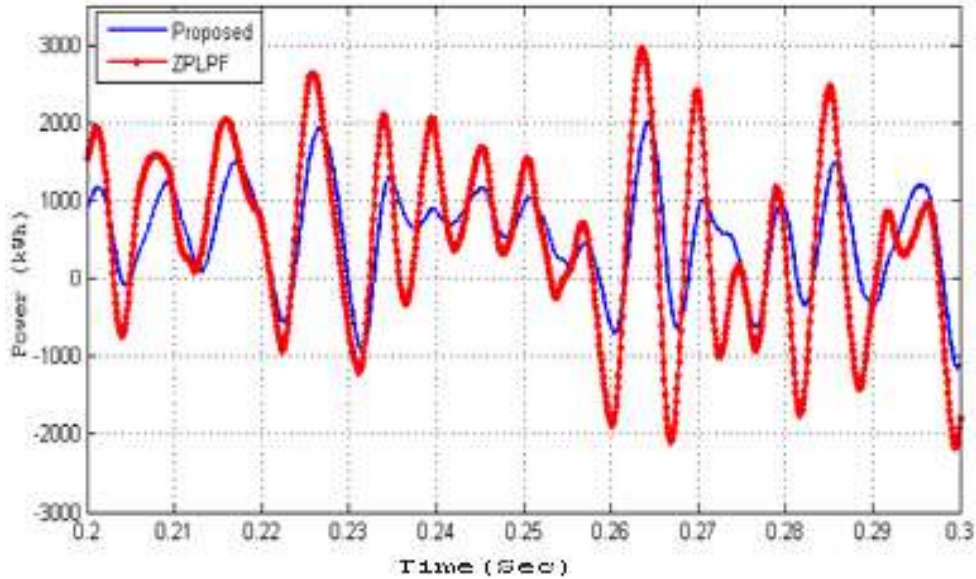

Figure 11. WT output power and output power delivered to the grid

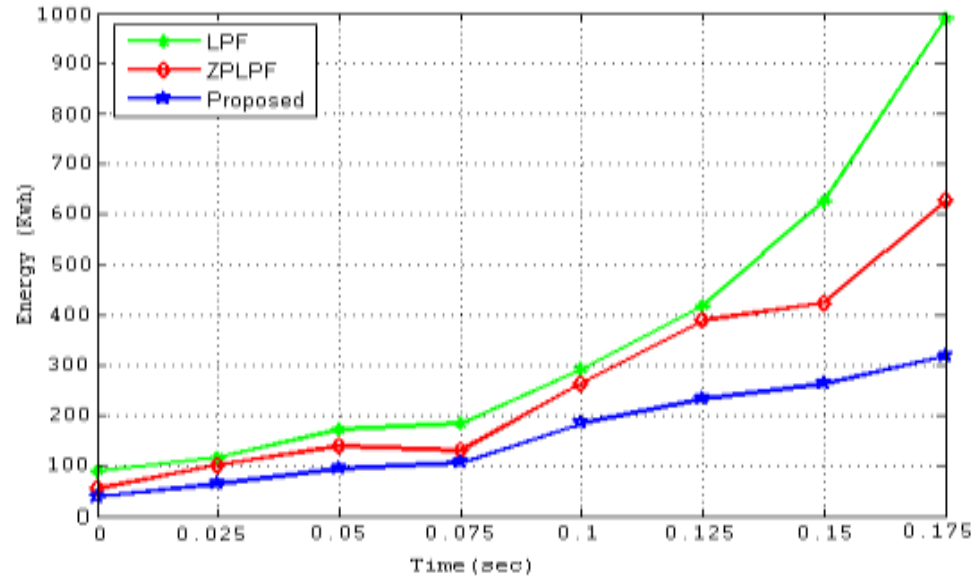

(a)

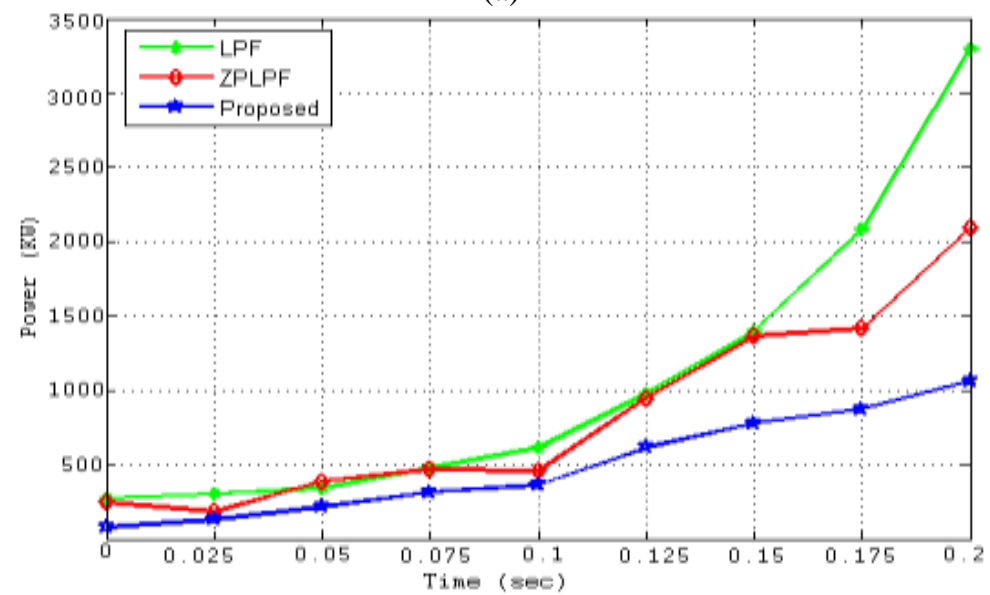

(b)

Figure 12. ESS capacity with respect time (a) Energy rating (b) Power rating 


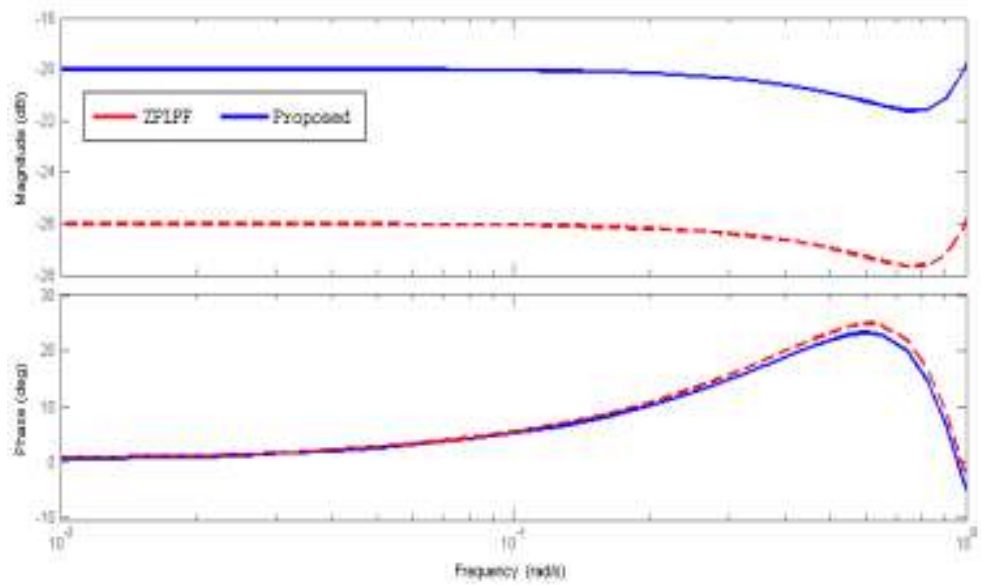

Figure 13. Bode diagram comparison for ZPLPF and proposed method

In Figure 12 (a) the energy rating of the existing LPF and ZPLPF are higher than that of the OZPLPF filter, in which at time $t=0.175 \mathrm{sec}$ the energy rating of the LPF, ZPLPF, OZPLPF are $100 \mathrm{kwh}, 610 \mathrm{kwh}, 310 \mathrm{kwh}$ respectively. Similarly, the power rating is shown in Figure 12 (b) in which the ESS is very much lesser for the proposed OZPLPF filter when comparing it with the existing LPF and ZPLPF. For instance, at time $\mathrm{t}=0.025 \mathrm{sec}$, the power hold by the LPF, ZPLPF, OZPLPF is of about $350 \mathrm{kw}, 200 \mathrm{kw}, 100 \mathrm{kw}$ respectively. Therefore, the ESS energy and power rating should be low for the optimal power dispatch, so that the expense will also be got reduced. Then, the comparison of bode diagram for the proposed and the conventional technique at constant smoothing time is made in Figure 13. From the above all analysis, the results shows that the proposed method requires a minimal-capacity ESS for dispatching wind power rather than the conventional methods.

\section{Conclusion}

This paper proposed an OZPLPF based wind power dispatch optimization with an ESS to overcome the non-adaptive performances of the conventional methods. The proposed dispatching model optimizes ZPLPF coefficients for various wind speed conditions based on the SNR. The suggested dispatching method has adaptive filter coefficients, robust model, easy to implement and eliminate the phase delay to reduce the capacity of the ESS. Therefore, the proposed method represents a feasible and optimal solution for dispatching wind power into the grid. The effectiveness of the proposed method has been analyzed by using the comparison analysis with the conventional methods. The results prove that, at any attenuation levels or on any the seasons, the proposed dispatching method achieves a much smaller ESS capacity compared with the conventional method.

\section{Reference}

[1]. Eftichios Koutroulis and Kostas Kalaitzakis, "Design of a Maximum Power Tracking System for Wind-Energy-Conversion Applications", IEEE Transactions on Industrial Electronics, Vol.53, No.2, pp.486-494, 2006

[2]. Nishad Mendis, Kashem M Muttaqi, Saad Sayeef and Sarath Perera, "Standalone Operation of Wind Turbine-Based Variable Speed Generators with Maximum Power Extraction Capability", IEEE Transactions on Energy Conversion, Vol.27, No.4, pp.822834, 2012

[3]. D Q Dang, Y Wang and W Cai, "Offset-Free Predictive Control for Variable Speed Wind Turbines”, IEEE Transactions on Sustainable Energy, Vol.4, No.1, pp.2-10, 2013 
[4]. Vivek Agarwal, Rakesh K Aggarwal, Pravin Patidar and Chetan Patki, "A Novel Scheme for Rapid Tracking of Maximum Power Point in Wind Energy Generation Systems", IEEE Transactions on Energy Conversion, Vol.25, No.1, pp.228-236, 2010

[5]. Zakariya M Dalala, Zaka Ullah Zahid and Jih-Sheng Lai, "New Overall Control Strategy for Small-Scale WECS in MPPT and Stall Regions with Mode Transfer Control", IEEE Transactions on Energy Conversion, Vol.28, No.4, pp.1082-1092, 2013

[6]. Maurizio Cirrincione, Marcello Pucci and Gianpaolo Vitale, "Growing Neural Gas-Based MPPT of Variable Pitch Wind Generators with Induction Machines”, IEEE Transactions on Industry Applications, Vol.48, No.3, pp.1006-1016, 2012

[7]. Jiawei Chen, Jie Chen and Chunying Gong, "New Overall Power Control Strategy for Variable-Speed Fixed-Pitch Wind Turbines Within the Whole Wind Velocity Range", IEEE Transactions on Industrial Electronics, Vol.60, No.7, pp.2652-2660, 2013

[8]. Jie Chen, Jiawei Chen, and Chunying Gong, "Constant-Bandwidth Maximum Power Point Tracking Strategy for Variable-Speed Wind Turbines and Its Design Details", IEEE Transactions on Industrial Electronics, Vol.60, No.11, pp.5050-5058, 2013

[9]. Yuanye Xia, Khaled H Ahmed and Barry W Williams, "Wind Turbine Power Coefficient Analysis of a New Maximum Power Point Tracking Technique", IEEE Transactions on Industrial Electronics, Vol.60, No.3, pp.1122-1132, 2013

[10]. Yuanye Xia, Khaled H Ahmed and Barry W Williams, "A New Maximum Power Point Tracking Technique for Permanent Magnet Synchronous Generator Based Wind Energy Conversion System", IEEE Transactions on Power Electronics, Vol.26, No.12, pp.36093620,2011

[11]. Kuo-Yuan Lo, Yaow-Ming Chen and Yung-Ruei Chang, "MPPT Battery Charger for Stand-Alone Wind Power System", IEEE Transactions on Power Electronics, Vol.26, No.6, pp.1631-1638, 2011

[12]. Athanasios Mesemanolis, Christos Mademlis and Iordanis Kioskeridis, "High-Efficiency Control for a Wind Energy Conversion System with Induction Generator", IEEE Transactions on Energy Conversion, Vol.27, No.4, pp.958-967, 2012

[13]. Yu Zou, Malik E Elbuluk and Yilmaz Sozer, "Stability Analysis of Maximum Power Point Tracking (MPPT) Method in Wind Power Systems", IEEE Transactions on Industry Applications, Vol.49, No.3, pp.1129-1136, 2013

[14]. Ching-Tsai Pan and Yu-Ling Juan, "A Novel Sensorless MPPT Controller for a HighEfficiency Microscale Wind Power Generation System”, IEEE Transactions on Energy Conversion, Vol.25, No.1, pp.207-216, 2010

[15]. Bharani Kumar Ramasamy, Aravindan Palaniappan and Sanavullah Mohamed Yakoh, "Direct-drive low-speed wind energy conversion system incorporating axial-type permanent magnet generator and Z-source inverter with sensorless maximum power point tracking controller”, IET Renewable Power Generation, Vol.7, No.3, pp.284-295, 2013

[16]. M Azzouz, A I Elshafei and H Emara, "Evaluation of fuzzy-based maximum powertracking in wind energy conversion systems", IET Renewable Power Generation, Vol.5, No.6, pp.422-430, 2011

[17]. Syed Muhammad Raza Kazmi, Hiroki Goto, Hai-Jiao Guo and Osamu Ichinokura, “A Novel Algorithm for Fast and Efficient Speed-Sensorless Maximum Power Point Tracking in Wind Energy Conversion Systems", IEEE Transactions On Industrial Electronics, Vol.58, No.1, pp.29-36, 2011

[18]. V Calderaro, V Galdi, A Piccolo, P Siano, “A fuzzy controller for maximum energy extraction from variable speed wind power generation systems", Electric Power Systems Research, Vol.78, No.6, pp.1109-1118, 2008

[19]. R Kot, M Rolak and M Malinowski, "Comparison of maximum peak power tracking algorithms for a small wind turbine", Mathematics and Computers in Simulation, Vol.91, pp.29-40, 2013 
[20]. Abdullah M A, Yatim A H M, Tan C W and Saidur R, "A review of maximum power point tracking algorithms for wind energy systems", Renewable and Sustainable Energy Reviews, Vol.16, No.5, pp.3220-3227, 2012

[21]. Hany M Jabr, Dongyun Lu and Narayan C Kar, "Design and Implementation of NeuroFuzzy Vector Control for Wind-Driven Doubly-Fed Induction Generator", IEEE Transactions on Sustainable Energy, Vol.2, No.4, pp.404-413, 2011

[22]. A Meharrar, M Tioursi, M Hatti and A Boudghène Stambouli, "A variable speed wind generator maximum power tracking based on adaptative neuro-fuzzy inference system", Expert Systems with Applications, Vol.38, No.6, pp.7659-7664, 2011

[23]. M Narayana, G A Putrus, M Jovanovic, P S Leung and S McDonald, "Generic maximum power point tracking controller for small-scale wind turbines", Renewable Energy, Vol.44, pp.72-79, 2012

[24]. Whei-Min Lin, Chih-Ming Hong, Cong-Hui Huang and Ting-Chia Ou, "Hybrid Control of a Wind Induction Generator Based on Grey-Elman Neural Network", IEEE Transactions on Control Systems Technology, Vol.21, No.6, pp.2367-2373, 2013

[25]. Ali M Eltamaly and Hassan M Farh, "Maximum power extraction from wind energy system based on fuzzy logic control", Electric Power Systems Research, Vol.97, pp.144150,2013

[26]. Juan P.Torreglosa, Pablo Garcia, Luis M. Fernandez and Francisco Jurado, "Energy dispatching based on predictive controller of an off-grid wind turbine/photovoltaic/hydrogen/battery hybrid system", Renewable Energy, Vol.74, pp.326-336, February 2015

[27]. Juan Pablo Saenz, Nurcin Celik, Hui Xi, Young-Jun Son and Shihab Asfour, "Two-stage economic and environmental load dispatching framework using particle filtering", International Journal of Electrical Power \& Energy Systems, Vol.48, pp.93-110, June 2013

[28]. H.Li, and Z.Chen, "Overview of different wind Generator Systems and Their Comparisons," IET Renewable Power Generation, Vol.2, No. 2, pp.123-138, June 2008

[29]. Cong-Long Nguyen and Hong-Hee Lee, "Optimization of Wind Power Dispatch to Minimize Energy Storage System Capacity", Journal of Electrical Engineering and Technology, Vol.9, No.3, pp. 1080-1088, 2014

[30]. Xiaojuan Han, Fang Chen, Xiwang Cui, Yong Li and Xiangjun Li, "A Power Smoothing Control Strategy and Optimized Allocation of Battery Capacity Based on Hybrid Storage Energy Technology", Energies, Vol.5, pp.1593-1612, 2012

[31]. Cong-Long Nguyen, Hyung-JunKim, Tay-Seek Lee and Hong-Hee Lee, "An Effective Dispatching Power Control to Minimize Energy Storage System in Wind Farm", In Proceedings of International Smart Grid Conference \& Exhibition 2013

[32]. Yasuhiro Hamada, Kiyotaka Takeda, Ryuichiro Goto and Hideki Kubota, "Hybrid utilization of renewable energy and fuel cells for residential energy systems", Energy and Buildings, Vol.43, No.12, pp.3680-3684, December 2011

[33]. Dervis Karaboga and Bahriye Basturk, "Artificial Bee Colony (ABC) Optimization Algorithm for Solving Constrained Optimization Problems", World Congress on Foundations of Fuzzy Logic and Soft Computing, pp.789-798, 2007

[34]. Parmjeet Kaur and Poonam Sethi, "Removal of Blocking Artifacts using Various Filtering Techniques", International Journal of Computer Applications, Vol.48, No.24, pp.11-18, June 2012 